Arq. Bras. Med. Vet. Zootec., v.68, n.2, p.369-378, 2016

\title{
Anestesia geral inalatória ou total intravenosa associada à anestesia subaracnoidea, em ovinos
}

\author{
[General inhalation anesthesia or total intravenous associated with \\ subarachnoid anesthesia in sheep]
}

\author{
M.P.A. Lima, A.L. Dallabrida, A.N. Moraes, M.I. Gehrcke, B.D.C. Regalin, \\ D. Regalin, F. Comassetto, N. Oleskovicz
}

Programa de Pós-Graduação - Centro de Ciências Agroveterinárias - Universidade do Estado de Santa Catarina (CAV-Udesc) - Lages, SC

\begin{abstract}
RESUMO
Avaliou-se a eficácia e a segurança anestésica em ovinos mantidos sob anestesia geral inalatória com isofluorano ou anestesia total intravenosa com propofol, ambas associadas à anestesia subaracnoidea. Quatorze ovinos foram pré-medicados com $0,3 \mathrm{mg} \cdot \mathrm{kg}^{-1}$ de morfina IM, e cinco minutos após, receberam $20 \mathrm{mcg} \cdot \mathrm{kg}^{-1} \mathrm{de}$ detomidina IV. Posteriormente, foram alocados aleatoriamente em dois grupos: GISO ( $\mathrm{n}=7$ ), os quais foram induzidos à anestesia geral com $0,5 \mathrm{mg} \cdot \mathrm{kg}^{-1}$ de diazepam e $5 \mathrm{mg} \cdot \mathrm{kg}^{-1}$ de cetamina IV, e mantidos em anestesia geral inalatória com isoflurano diluído em oxigênio a 100\%; e GPRO $(\mathrm{n}=7)$, induzidos com $4 \mathrm{mg}^{\mathrm{kg}} \mathrm{kg}^{-1} \mathrm{de}$ propofol IV seguido inicialmente de infusão contínua na taxa de $0,3 \mathrm{mg} \cdot \mathrm{kg}^{-1} \cdot \mathrm{min}^{-1}$. Para realização da osteotomia bilateral, todos os animais receberam $0,5 \mathrm{mg} \cdot \mathrm{kg}^{-1}$ de ropivacaína $0,75 \%$ associado a $0,1 \mathrm{mg}^{-\mathrm{kg}^{-1}} \mathrm{de}$ morfina pela via subaracnoidea. Houve redução de $40 \%$ nos valores médios de frequência cardíaca após sedação em ambos os grupos, permanecendo em média $23 \%$ reduzida até o final da avaliação. A pressão arterial média aumentou $16 \%$, após a indução anestésica no GISO, mas se reduziu até o final do procedimento, assim como no GPRO. A EtISO média foi de $0,57 \mathrm{~V} \%$ e a taxa média de infusão do propofol foi de $0,24 \mathrm{mg} \cdot \mathrm{kg}^{-}$ ${ }^{1} . \mathrm{min}^{-1}$. Os tempos totais de cirurgia, anestesia e extubação foram de $66 \pm 9,8,92 \pm 13,8$ e 7,0 $\pm 1,5$ minutos no GISO e $56 \pm 2,4,82,9 \pm 4,6$ e 5,4 $\pm 1,5$ minutos no GPRO, não havendo diferença significativa entre grupos. A manutenção anestésica com isoflurano ou propofol promoveu plano anestésico similar com mínimos efeitos cardiovasculares ou hemogasométricos, que são bem tolerados em ovinos hígidos.
\end{abstract}

Palavras-chave: anestesia balanceada, propofol, isoflurano, ropivacaína

\begin{abstract}
The purpose of this study was to evaluate the efficacy and safety of sheep submitted to inhalation anesthesia with isoflurane or total intravenous anesthesia with propofol, both associated with subarachnoid anesthesia. Fourteen animals were pre-medicated with $0.3 \mathrm{mg} . \mathrm{kg}^{-1}$ morphine IM, and 5 minutes later received $20 \mathrm{mcg} . \mathrm{kg}^{-1}$ detomidine IV. Then they were allocated into two groups: GISO $(\mathrm{n}=7)$, which were induced with $0.5 \mathrm{mg} . \mathrm{kg}^{-1}$ of diazepam and $5 \mathrm{mg}_{\mathrm{kg}}{ }^{-1}$ of ketamine IV, and anesthesia maintenance was performed by isoflurane diluted in $100 \%$ oxygen; or GPRO ( $(n=7)$, where animals were induced with $4 \mathrm{mg} . \mathrm{kg}^{-1}$ propofol IV and subsequent maintenance anesthesia with its own infusion of $0.3 \mathrm{mg} \cdot \mathrm{kg} \cdot \mathrm{min}^{-1}$. To perform the bilateral tibial osteotomy, all animals received $0.5 \mathrm{mg} \cdot \mathrm{kg}^{-1} 0.75 \%$ ropivacaine combined with $0.1 \mathrm{mg} \cdot \mathrm{kg}^{-1}$ morphine by the intrathecal route. There was a $40 \%$ reduction in mean heart rate after the sedative protocol in both groups, resulting in a $23 \%$ average reduction until the end of the review. Mean arterial pressure showed transient elevation of around $16 \%$, after induction of anesthesia in GISO, but reducing it to the end of the procedure, as well as in GPRO. The average EtISO was $0.57 \mathrm{~V} \%$ and average infusion rate of propofol was $0.24 \mathrm{mg} \cdot \mathrm{kg}^{-1} . \mathrm{min}^{-1}$. The total time of surgery, anesthesia and extubation was $66 \pm 9.8,92 \pm 7.0$ and $13.8 \pm 1.5$ minutes in GISO and 56 $\pm 2.4,82.9 \pm 4.6$ and 5.4 \pm 1.5 minutes in GPRO. The maintenance of anesthesia with propofol or isoflurane produced similar anesthesia with minimal cardiovascular and blood gas effects, which are well tolerated in healthy sheep.
\end{abstract}

Keywords: balanced anesthesia, propofol, isoflurane, ropivacaine

Recebido em 22 de setembro de 2014

Aceito em 15 de novembro de 2015

E-mail: limampa@hotmail.com 


\section{INTRODUÇÃO}

O número de intervenções cirúrgicas realizadas na espécie ovina vem aumentando, principalmente em animais de alto valor zootécnico (Lizarraga e Chambers, 2012). Além disso, os pequenos ruminantes se destacam como um dos modelos experimentais mais adotados para cirurgias torácicas, cardíacas e ortopédicas por apresentarem tamanho considerado adequado, proporcionarem maior facilidade de manuseio comparados a caninos e suínos, serem de fácil obtenção e por apresentarem respostas hemodinâmicas farmacológicas semelhantes ao homem (Adams e Mckinley, 2009).

Dentre as técnicas para manutenção anestésica, destacam-se a anestesia inalatória e a anestesia total intravenosa (ATI), que vem ganhando espaço na medicina veterinária como alternativa à anestesia inalatória, pois produz plano anestésico adequado, não polui o ambiente cirúrgico e não requer aparelhagem específica quando comparada à anestesia inalatória. Para tal, o propofol é indicado por ser um fármaco anestésico intravenoso de curta duração, associado a mínimos efeitos excitatórios e recuperação anestésica suave, rápido metabolismo extra-hepático (Correia et al., 1996). Contudo, a anestesia inalatória promove melhor controle do plano anestésico devido a rápidas alterações na concentração plasmática, requer mínima metabolização dos agentes, visto que estes são excretados inalterados via pulmonar, e promove menor efeito cumulativo mesmo após longos períodos de anestesia.

A anestesia subaracnoidea propicia a realização de procedimentos que envolvam a região da cauda, o períneo e os membros pélvicos, bem como animais submetidos à laparotomia retroumbilical. Diversos fármacos são empregados para a realização dessa técnica por apresentarem propriedades anestésicas e/ou analgésicas, o que reduz o requerimento do agente hipnótico empregado e proporciona a manutenção anestésica em planos mais superficiais (Lizarraga e Chamber, 2012).

O objetivo deste estudo foi avaliar os efeitos cardiovasculares e respiratórios da manutenção anestésica com anestesia geral inalatória com isoflurano, ou infusão contínua de propofol, associada à anestesia subaracnoidea com ropivacaína e morfina, em ovinos submetidos à osteotomia bilateral de tíbia.

\section{MATERIAL E MÉTODOS}

Este estudo foi aprovado pelo Comitê de Ética e Bem-Estar Animal (CETEA) da instituição de origem (Protocolo 1.70/12). Foram utilizados 14 ovinos, mestiços, adultos, fêmeas, com peso médio de $27 \pm 3,5 \mathrm{~kg}$, classificados como pacientes ASA I, confirmado por meio de exame clínico e de exames complementares, como hemograma, função renal e função e lesão hepática. Os animais foram everminados e mantidos durante 20 dias em baias coletivas, alimentados com ração balanceada e silagem de milho, duas vezes ao dia, com acesso a piquetes de pastagens ao longo do dia e água ad libitum. Previamente ao início do estudo, passaram por jejum alimentar e hídrico de 24 e 12 horas, respectivamente.

No dia do estudo, realizou-se preparação dos animais, que consistiu na inserção de cateter intravenoso central Intracath $20 \mathrm{G}$ na veia jugular esquerda, acesso venoso da veia cefálica esquerda com cateter $18 \mathrm{G}$ e acesso da artéria marginal da orelha esquerda com cateter $22 \mathrm{G}$. Em seguida, com o animal em decúbito lateral direito, foram aferidos os parâmetros basais: frequência cardíaca $(\mathrm{FC})$, em batimentos por minuto (bpm), por meio de oximetria de pulso; frequência respiratória $(f)$, em movimentos respiratórios por minutos (mpm), por monitor de capnógrafo; temperatura corporal (TC), em graus Celsius, por meio de termômetro esofágico; pressão arterial média (PAM), em $\mathrm{mm} / \mathrm{Hg}$, por meio de leitura em monitor multiparamétrico; pressão venosa central (PVC), em $\mathrm{cm} / \mathrm{H}_{2} \mathrm{O}$, e análise hemogasométrica do sangue arterial (iSTAT ${ }^{\circledR}$ Portable handheld, Abbott Point of Care, Princenton, NJ, EUA), obtendo-se: valores de potencial de hidrogênio $(\mathrm{pH})$; pressão parcial de dióxido de carbono $\left(\mathrm{PaCO}_{2}\right)$, em $\mathrm{mm} / \mathrm{Hg}$; pressão parcial de oxigênio $\left(\mathrm{PaO}_{2}\right)$, em $\mathrm{mm} / \mathrm{Hg}$; excesso de base no líquido extracelular (BEecf), bicarbonato $\left(\mathrm{HCO}^{-3}\right)$, todos em $\mathrm{mmol} / \mathrm{L}$; saturação de oxi-hemoglobina $\left(\mathrm{SaO}_{2}\right)$, em \%; glicemia, em mg/dL.

$\mathrm{Na}$ sequência, todos os animais receberam $0,3 \mathrm{mg} \cdot \mathrm{kg}^{-1}$ de morfina pela via intramuscular (IM) e, cinco minutos após, receberam 20 mcg. $\mathrm{kg}^{-1}$ de detomidina pela via intravenosa (IV). Nesse momento, foram alocados 
aleatoriamente em dois grupos: grupo isoflurano (GISO), o qual foi induzido à anestesia geral com $0,5 \mathrm{mg} \cdot \mathrm{kg}^{-1}$ de diazepam e $5 \mathrm{mg} \cdot \mathrm{kg}^{-1}$ de cetamina IV e manutenção anestésica realizada com isoflurano inicialmente a $0,7 \mathrm{~V} \%$, diluído em oxigênio a 100\%; e grupo propofol (GPRO), o qual foi induzidos à anestesia geral com $4 \mathrm{mg} \cdot \mathrm{kg}^{-1}$ de propofol administrado em um minuto, por meio de bomba de infusão de seringa, e manutenção por meio de infusão contínua na taxa de $0,3 \mathrm{mg} \cdot \mathrm{kg}^{-1} \cdot \mathrm{min}^{-1}$, do momento da indução anestésica até 10 minutos após a realização da anestesia subaracnoidea, sendo então reduzida para $0,2 \mathrm{mg} \cdot \mathrm{kg}^{-1} \cdot \mathrm{min}^{-1}$.

O escore de sedação foi avaliado cinco minutos após a administração intravenosa de detomidina, de acordo com escala adaptada de Abu-Ahmed, 2013 e DeRossi et al., 2012, e a qualidade de indução anestésica e intubação orotraqueal de acordo com escala adaptada de Jud et al., 2010, conforme descritos na Tab.1.

Tabela 1. Escores de sedação, indução anestésica e intubação orotraqueal, em ovinos pré-medicados com morfina e detomidina, induzidos com propofol (GPRO) ou cetamina e diazepam (GISO), e mantidos sob infusão contínua de propofol (GPRO, n=7) ou anestesia geral inalatória com isoflurano (GISO, n=7)

\begin{tabular}{|c|c|c|c|}
\hline & Sedação* & Indução & Intubação \\
\hline Escore 1 & $\begin{array}{l}\text { Sedação intensa, } \\
\text { animal em decúbito } \\
\text { lateral, irresponsivo a } \\
\text { estímulos. }\end{array}$ & $\begin{array}{l}\text { (Excelente) Indução suave, } \\
\text { ausência de tremores musculares, } \\
\text { ausência de movimentação para } \\
\text { frente ou para trás, ausência de } \\
\text { excitação }\end{array}$ & $\begin{array}{l}\text { (Excelente) Intubação fácil } \\
\text { à primeira tentativa, bom } \\
\text { relaxamento muscular. }\end{array}$ \\
\hline Escore 2 & $\begin{array}{l}\text { Sedação moderada, } \\
\text { animal com } \\
\text { abaixamento de cabeça, } \\
\text { ptose palpebral, leve } \\
\text { ataxia. }\end{array}$ & $\begin{array}{l}\text { (Bom) Indução suave, presença de } \\
\text { leves tremores de cabeça ou } \\
\text { membros. }\end{array}$ & $\begin{array}{l}\text { (Bom) Intubação à segunda } \\
\text { tentativa, reflexos laríngeos } \\
\text { ou de deglutição levemente } \\
\text { presentes. }\end{array}$ \\
\hline Escore 3 & $\begin{array}{l}\text { Sedação leve, animal } \\
\text { menos alerta, ptose } \\
\text { palpebral, sem outros } \\
\text { sinais. }\end{array}$ & $\begin{array}{l}\text { (Razoável) Promove decúbito, mas } \\
\text { com pobre relaxamento muscular e } \\
\text { de membros, tentativa de levantar a } \\
\text { cabeça. }\end{array}$ & $\begin{array}{l}\text { (Razoável) Intubação à } \\
\text { terceira tentativa, reflexos } \\
\text { laríngeos ou de deglutição } \\
\text { presentes, pouco } \\
\text { relaxamento muscular. }\end{array}$ \\
\hline Escore 4 & $\begin{array}{l}\text { Ausência de efeitos } \\
\text { sedativos. }\end{array}$ & $\begin{array}{l}\text { (Ruim) Indução com extrema } \\
\text { movimentação, excitação e } \\
\text { tentativas de levantar do animal. }\end{array}$ & $\begin{array}{l}\text { (Ruim) Intubação realizada } \\
\text { após dose suplementar dos } \\
\text { fármacos indutores. }\end{array}$ \\
\hline
\end{tabular}

*Adaptado de Abu-Ahmed 2013 e DeRossi et al., 2012; " Adaptado de Jud et al., 2010.

Após a indução anestésica, todos os animais foram intubados com sonda endotraqueal de Murphy com cuff e mantidos sob ventilação mecânica limitada à pressão $\left(20 \mathrm{~cm} / \mathrm{H}_{2} \mathrm{O}\right)$, ajustando a frequência respiratória para manutenção da capnometria dentro de uma faixa de 35 a 40mmHg.Todos os animais foram submetidos à osteotomia bilateral de tíbia, em um estudo paralelo, produzindo-se defeitos ósseos na tíbia como modelo experimental para avaliação do comportamento da neoformação óssea e da osteointegração de hidroxiapatita (HA), tricálcio fosfato- $\beta$ (TCP- $\beta$ ), hidroxiapatita/tricálcio fosfato- $\beta$ (HA/TCP- $\beta$ 60/40) e o nano compósito hidroxiapatita/alumina a 5\% (HA/Al2O3 a 5\%), por isso receberam $0,5 \mathrm{mg} \cdot \mathrm{kg}^{-1}$ de ropivacaína $0,75 \%$ associado a $0,1 \mathrm{mg} \cdot \mathrm{kg}^{-1}$ de morfina pela via subaracnoidea, diluindo-se com solução $\mathrm{NaCl}$ $0,9 \%$, para um volume final de $1 \mathrm{~mL} / 7,5 \mathrm{~kg}$, administrados por meio de agulha espinhal 19G, no espaço lombossacro (L6-S1), sendo o volume aplicado em um minuto. Imediatamente após, os animais foram posicionados em decúbito dorsal e preparados para o ato cirúrgico.

Os parâmetros aferidos no momento basal foram novamente aferidos cinco minutos após a administração da detomidina (M1); cinco minutos após a indução anestésica (M2); 10 minutos após a realização da anestesia subaracnoidea e posicionados em decúbito dorsal (M3); imediatamente após a realização da osteotomia do membro pélvico direito (M4); 30 minutos após a realização da anestesia subaracnoidea em decúbito dorsal (M5); 
imediatamente após a realização da osteotomia do membro pélvico esquerdo (M6); e ao término da cirurgia/anestesia (M7), com exceção da avaliação hemogasométrica, que não foi avaliada nos momentos M4 e M6.

O plano anestésico foi mantido com considerando-se a avaliação de parâmetros clínicos, baseados nos estágios da anestesia propostos por Guedel, objetivando-se manter os animais com o globo ocular rotacionado, ausência do reflexo palpebral lateral, ausência ou discreta presença do reflexo palpebral medial, presença de reflexo corneal e parâmetros cardiorrespiratórios dentro do intervalo fisiológico para a espécie.

A EtISO, em volume \% (V\%), foi avaliada por meio da leitura direta no monitor do analisador de gases, cujo sensor foi adaptado entre a sonda endotraqueal e o sistema do aparelho de anestesia. Ao final da anestesia, o total de propofol infundido foi registrado, e calculada a dose média em infusão contínua empregada para manutenção.

Durante o ato cirúrgico, caso houvesse aumento de FC ou PAM em $20 \%$ em relação ao momento imediatamente anterior, administrava-se bolus de $5 \mathrm{mcg} . \mathrm{kg}^{-1}$ de fentanil IV, em dose suficiente para suprimir o processo álgico, retornando os valores à normalidade. Caso não houvesse resgastes analgésicos, considerava-se como eficaz o bloqueio subaracnoideo. Durante a recuperação anestésica, avaliou-se qualidade do retorno da anestesia, o tempo para extubação e a ocorrência de efeitos adversos.

O teste de Shapiro Wilk foi realizado para avaliar a ocorrência de distribuição normal dos dados. Para análise entre tempos dentro do mesmo grupo, utilizou-se ANOVA-RM, seguido pelo teste de Dunnet $(\mathrm{P} \leq 0,05)$. Para comparação entre os mesmos momentos dos diferentes grupos, empregou-se o teste $t$. Para avaliação dos dados não paramétricos, como qualidade de sedação, indução e intubação, foi usado o teste de Mann Whitney Rank Sum Test $(\mathrm{P} \leq 0,05)$.

\section{RESULTADOS}

O peso médio dos animais foi maior no GISO $(28,8 \pm 2,6 \mathrm{~kg})$ quando comparado ao GPRO $(24,7 \pm 2,9 \mathrm{~kg})$. Os tempos cirúrgico e anestésico foram semelhantes, sendo de $66,0 \pm 9,8$ e $92,0 \pm 13,8$ no GISO, e de $56,0 \pm 2,4$ e $82,9 \pm 4,6$ no GPRO, respectivamente. Não foram observadas diferenças significativas em relação aos escores de sedação e qualidade de indução anestésica, enquanto a facilidade de intubação orotraqueal foi significativamente maior no GPRO em relação ao GISO (Tab. 2).

Tabela 2. Porcentagem e proporção de animais, de acordo com o escore obtido, em relação à sedação, indução anestésica e intubação orotraqueal, em ovinos mantidos sob anestesia geral inalatória com isoflurano (GISO, $n=7$ ) ou infusão contínua de propofol (GPRO, $\mathrm{n}=7$ )

\begin{tabular}{lcccc} 
& Grupo & Sedação & Indução & Intubação \\
\hline \multirow{2}{*}{ Escore 1} & GISO & $0 / 7$ & $100 \%(7 / 7)$ & $57,1 \%(4 / 7)^{\mathrm{a}}$ \\
& GPRO & $0 / 7$ & $100 \%(7 / 7)$ & $85,7 \%(6 / 7)^{\mathrm{b}}$ \\
\multirow{2}{*}{ Escore 2} & GISO & $85,7 \%(6 / 7)$ & $0 / 7$ & $42,9 \%(3 / 7)^{\mathrm{a}}$ \\
& GPRO & $71,4 \%(5 / 7)$ & $0 / 7$ & $14,3 \%(1 / 7)^{\mathrm{b}}$ \\
\multirow{2}{*}{ Escore 3} & GISO & $14,3 \%(1 / 7)$ & $0 / 7$ & $0 / 7$ \\
& GPRO & $28,6 \%(2 / 7)$ & $0 / 7$ & $0 / 7$ \\
Escore 4 & GISO & $0 / 7$ & $0 / 7$ & $0 / 7$ \\
& GPRO & $0 / 7$ & $0 / 7$ & $0 / 7$ \\
\hline
\end{tabular}

Letras minúsculas diferentes nas colunas, dentro de cada variável, indicam valor diferente entre grupos. Mann Whitney Rank Sum Test $(\mathrm{P} \leq 0,05)$.

Salivação profusa foi observada em todos os animais do momento M1 até o M7, em ambos os grupos, e diurese após a administração da medicação pré-anestésica em cinco de sete animais do GISO e quatro de sete animais do GPRO.
Houve redução dos valores médios de FC de M1 a M7 quando comparados a M0, em ambos os grupos. Esta redução foi em média de $40 \%$ em M1, e manteve-se reduzida $23 \%$ até $\mathrm{M} 7$, em ambos os grupos. Os valores de FC em M2 
foram significativamente menores no GISO quando comparados ao GPRO (Tab. 3).

Os valores médios da PAM elevaram-se $16 \%$ em M2 quando comparado ao M0 e posteriormente reduziram de M4 a M7 no GISO. No GPRO, os valores médios dessa variável reduziram significativamente de M3 a M7 quando comparados ao M0. Entre os grupos estudados, observaram-se valores significativamente menores de PAM em M2 e M3 no GPRO quando comparados ao GISO (Tab. 3).

Os valores médios de PVC elevaram-se em M1 e M2 em relação ao M0, em ambos os grupos, sendo significativamente menores em M3 no GPRO quando comparado ao GISO. Os valores médios da TC reduziram de M3 a M7 quando comparados ao $\mathrm{M} 0$, em ambos os grupos, não havendo diferenças significativas entre grupos.

Tabela 3. Valores médios e desvio-padrão da frequência cardíaca (FC), frequência respiratória (f), pressão arterial média (PAM), pressão venosa central (PVC), temperatura corpórea (TC), em ovinos mantidos sob anestesia geral inalatória com isoflurano (GISO, $\mathrm{n}=7$ ) ou infusão contínua de propofol $(\mathrm{GPRO}, \mathrm{n}=7$ )

\begin{tabular}{|c|c|c|c|c|c|c|c|c|c|}
\hline & \multirow[t]{2}{*}{ Grupos } & \multicolumn{8}{|c|}{ Momentos } \\
\hline & & M0 & M1 & M2 & M3 & M4 & M5 & M6 & M7 \\
\hline \multirow[t]{2}{*}{$\mathrm{FC}$} & GISO & 114 & $68 \mathrm{~A}$ & $74 \mathrm{Aa}$ & $84 \mathrm{~A}$ & $91 \mathrm{~A}$ & $92 \mathrm{~A}$ & $89 \mathrm{~A}$ & $87 \mathrm{~A}$ \\
\hline & & \pm 12 & \pm 6 & \pm 6 & \pm 15 & \pm 11 & \pm 11 & \pm 9 & \pm 9 \\
\hline \multirow[t]{2}{*}{ (bpm) } & GPRO & 119 & $73 \mathrm{~A}$ & $86 \mathrm{Ab}$ & $93 \mathrm{~A}$ & $91 \mathrm{~A}$ & $88 \mathrm{~A}$ & $84 \mathrm{~A}$ & $83 \mathrm{~A}$ \\
\hline & & \pm 18 & \pm 13 & \pm 11 & \pm 13 & \pm 13 & \pm 13 & \pm 12 & \pm 17 \\
\hline \multirow[t]{2}{*}{$f$} & GISO & 27 & 24 & 31 & $9 \mathrm{~A}$ & $8 \mathrm{~A}$ & $8 \mathrm{~A}$ & $8 \mathrm{~A}$ & $8 \mathrm{~A}$ \\
\hline & & \pm 4 & \pm 4 & \pm 16 & \pm 1 & \pm 0 & \pm 0 & \pm 0 & \pm 0 \\
\hline \multirow[t]{2}{*}{ (mpm) } & GPRO & 28 & 25 & 26 & $8 \mathrm{~A}$ & $8 \mathrm{~A}$ & 7A & $7 \mathrm{~A}$ & $7 \mathrm{~A}$ \\
\hline & & \pm 3 & \pm 5 & \pm 8 & \pm 1 & \pm 1 & \pm 1 & \pm 1 & \pm 1 \\
\hline \multirow[t]{2}{*}{ PAM } & GISO & 95 & 95 & $110 \mathrm{Aa}$ & $92 a$ & $80 \mathrm{~A}$ & $79 \mathrm{~A}$ & $78 \mathrm{~A}$ & $81 \mathrm{~A}$ \\
\hline & & \pm 11 & \pm 8 & \pm 13 & \pm 10 & \pm 11 & \pm 11 & \pm 9 & \pm 11 \\
\hline \multirow[t]{2}{*}{$(\mathrm{mmHg})$} & GPRO & 90 & 93 & $96 b$ & $79 \mathrm{Ab}$ & $75 \mathrm{~A}$ & $72 \mathrm{~A}$ & $73 \mathrm{~A}$ & 77A \\
\hline & & \pm 2 & \pm 9 & \pm 11 & \pm 6 & \pm 7 & \pm 8 & \pm 7 & \pm 10 \\
\hline \multirow[t]{2}{*}{ PVC } & GISO & 0,6 & $7,7 \mathrm{~A}$ & $6,6 \mathrm{~A}$ & $2,0 \mathrm{a}$ & $-0,3$ & $-0,9$ & $-2,4$ & $-2,1$ \\
\hline & & $\pm 3,5$ & $\pm 1,5$ & $\pm 1,4$ & $\pm 4,7$ & $\pm 3,7$ & $\pm 3,5$ & $\pm 3,9$ & $\pm 4,3$ \\
\hline \multirow[t]{2}{*}{$\left(\mathrm{cmH}_{2} \mathrm{O}\right)$} & GPRO & $-0,6$ & $7,7 \mathrm{~A}$ & $5,1 \mathrm{~A}$ & $-2,7 b$ & $-2,7$ & $-3,3$ & $-3,1$ & $-3,6$ \\
\hline & & $\pm 3,2$ & $\pm 3,0$ & $\pm 3,6$ & $\pm 1,6$ & $\pm 1,6$ & $\pm 2,1$ & $\pm 1,9$ & $\pm 1,5$ \\
\hline \multirow[t]{2}{*}{$\mathrm{TC}$} & GISO & 38,7 & 38,9 & 38,5 & $37,6 \mathrm{~A}$ & $37,3 \mathrm{~A}$ & $37,2 \mathrm{~A}$ & $36,7 \mathrm{~A}$ & $36,5 \mathrm{~A}$ \\
\hline & & $\pm 0,6$ & $\pm 0,6$ & $\pm 0,7$ & $\pm 0,5$ & $\pm 0,4$ & $\pm 0,5$ & $\pm 0,6$ & $\pm 0,6$ \\
\hline \multirow[t]{2}{*}{$\left({ }^{\circ} \mathrm{C}\right)$} & GPRO & 38,7 & 38,8 & 38,3 & $37,7 \mathrm{~A}$ & $37,4 \mathrm{~A}$ & $37,2 \mathrm{~A}$ & $36,9 \mathrm{~A}$ & $37,0 \mathrm{~A}$ \\
\hline & & $\pm 0,4$ & $\pm 0,4$ & $\pm 0,2$ & $\pm 0,2$ & $\pm 0,3$ & $\pm 0,2$ & $\pm 0,2$ & $\pm 0,7$ \\
\hline
\end{tabular}

Letra A na linha indica valor diferente de M0, teste de Dunnet $(\mathrm{P} \leq 0,05)$. Letras minúsculas diferentes nas colunas, dentro de cada momento, indicam valor diferente entre grupos, Teste $t(\mathrm{P} \leq 0,05)$.

Como demonstrado na Tab. 4, foi observada ocorrência de alcalose metabólica observada de M3 a M7, em ambos os grupos, caracterizada por elevação do $\mathrm{pH}$, acúmulo de bicarbonato, elevação do EB, quando comparados com os valores basais e diante de valores de $\mathrm{PaCO}_{2}$ normais. Houve aumento significativo dos valores médios de $\mathrm{PaO}_{2}$ e $\mathrm{SaO}_{2}$ de $\mathrm{M} 2$ a $\mathrm{M} 7$ em comparação ao M0, em ambos os grupos, não havendo diferenças significativas entre grupos. Os níveis séricos de $\mathrm{K}^{+}$reduziram-se de $\mathrm{M} 2$ a M7, em ambos os grupos, enquanto os níveis glicêmicos elevaram-se de M1 a M7, sendo significativamente maiores em M1 no GISO quando comparado ao GPRO.
A EtISO média necessária para manutenção do plano anestésico foi de $0,57 \pm 0,1 \mathrm{~V} \%$, enquanto a taxa média de infusão de propofol foi de $0,24 \pm 0,11 \mathrm{mg} \cdot \mathrm{kg}^{-1} \cdot \mathrm{min}^{-1}$.

Considerou-se o bloqueio subarancoide como eficaz em $100 \%$ dos animais de ambos os grupos, pois alterações cardiovasculares não foram observadas durante os momentos de incisão de pele, do periósteo e da realização das osteotomias, considerado o estímulo supramáximo do procedimento cirúrgico, não requerendo resgates analgésicos. 
Tabela 4. Valores médios e desvio-padrão dos valores do potencial de hidrogênio $(\mathrm{pH})$, pressão parcial de dióxido de carbono arterial $\left(\mathrm{PaCO}_{2}\right)$, pressão parcial de oxigênio arterial $\left(\mathrm{PaO}_{2}\right)$, excesso de base (BEecf), bicarbonato $\left(\mathrm{HCO}_{3}^{-}\right)$, saturação de oxiemoglobina $\left(\mathrm{SaO}_{2}\right)$, níveis glicêmicos, no sangue arterial, em ovinos mantidos sob anestesia geral inalatória com isoflurano (GISO, n=7) ou infusão contínua de propofol (GPRO, $\mathrm{n}=7$ )

\begin{tabular}{|c|c|c|c|c|c|c|c|}
\hline & \multirow[t]{2}{*}{ Grupos } & \multicolumn{6}{|c|}{ MOMENTOS } \\
\hline & & M0 & M1 & M2 & M3 & M5 & M7 \\
\hline \multirow[t]{4}{*}{$\mathrm{pH}$} & GISO & 7,45 & 7,44 & $7,33 \mathrm{~A}$ & 7,49 & 7,47 & 7,48 \\
\hline & & $\pm 0,04$ & $\pm 0,05$ & $\pm 0,05$ & $\pm 0,04$ & $\pm 0,02$ & $\pm 0,02$ \\
\hline & GPRO & 7,44 & 7,44 & $7,34 \mathrm{~A}$ & 7,49 & $7,49 \mathrm{~A}$ & 7,49 \\
\hline & & $\pm 0,03$ & $\pm 0,04$ & $\pm 0,02$ & $\pm 0,05$ & $\pm 0,06$ & $\pm 0,05$ \\
\hline \multirow{2}{*}{$\mathrm{PaCO}_{2}$} & GISO & 33,50 & 36,60 & $53,06 \mathrm{~A}$ & $40,76 \mathrm{~A}$ & $42,41 \mathrm{~A}$ & $43,56 \mathrm{~A}$ \\
\hline & & $\pm 1,9$ & $\pm 2,22$ & $\pm 6,44$ & $\pm 4,50$ & $\pm 3,80$ & $\pm 4,23$ \\
\hline \multirow[t]{2}{*}{$(\mathrm{mmHg})$} & GPRO & 36,17 & 39,04 & $50,73 \mathrm{~A}$ & $41,33 \mathrm{~A}$ & $41,94 \mathrm{~A}$ & $44,57 \mathrm{~A}$ \\
\hline & & $\pm 2,04$ & $\pm 3,00$ & $\pm 3,32$ & $\pm 4,02$ & $\pm 4,67$ & $\pm 3,68$ \\
\hline \multirow[t]{2}{*}{$\mathrm{PaO}_{2}$} & GISO & 72,68 & 69,64 & $331,18 \mathrm{~A}$ & $364,62 \mathrm{~A}$ & $343,43 \mathrm{~A}$ & $361,62 \mathrm{~A}$ \\
\hline & & $\pm 10,66$ & $\pm 4,92$ & $\pm 25,82$ & $\pm 24,01$ & $\pm 37,32$ & $\pm 27,72$ \\
\hline \multirow[t]{2}{*}{$(\mathrm{mmHg})$} & GPRO & 80,28 & 71 & $315,86 \mathrm{~A}$ & $330,43 \mathrm{~A}$ & $340,43 \mathrm{~A}$ & $359,43 \mathrm{~A}$ \\
\hline & & $\pm 15,71$ & $\pm 4,51$ & $\pm 26,62$ & $\pm 50,41$ & $\pm 42,41$ & $\pm 37,97$ \\
\hline \multirow[t]{2}{*}{ BEecf } & GISO & $-0,43$ & 1,00 & $1,86 \mathrm{~A}$ & $7,29 \mathrm{~A}$ & $6,86 \mathrm{~A}$ & $8,71 \mathrm{~A}$ \\
\hline & & $\pm 3,31$ & $\pm 3,11$ & $\pm 3,19$ & $\pm 1,89$ & $\pm 2,55$ & $\pm 3,73$ \\
\hline \multirow[t]{2}{*}{$(\mathrm{mEq} / \mathrm{L})$} & GPRO & 0,58 & 2,86 & $4,86 \mathrm{~A}$ & $8,28 \mathrm{~A}$ & $9,14 \mathrm{~A}$ & $9,71 \mathrm{~A}$ \\
\hline & & $\pm 2,94$ & $\pm 2,79$ & $\pm 1,68$ & $\pm 3,09$ & $\pm 2,85$ & $\pm 3,15$ \\
\hline \multirow[t]{2}{*}{$\mathrm{HCO}_{3}^{-}$} & GISO & 23,13 & 24,86 & $27,58 \mathrm{~A}$ & $30,67 \mathrm{~A}$ & $30,40 \mathrm{~A}$ & $32,46 \mathrm{~A}$ \\
\hline & & $\pm 2,79$ & $\pm 3,26$ & $\pm 3,54$ & $\pm 1,90$ & $\pm 2,59$ & $\pm 3,50$ \\
\hline \multirow[t]{2}{*}{$(\mathrm{mmol} / \mathrm{L})$} & GPRO & 24,41 & 26,43 & $29,47 \mathrm{~A}$ & $31,30 \mathrm{~A}$ & $32,28 \mathrm{~A}$ & $33,29 \mathrm{~A}$ \\
\hline & & $\pm 2,48$ & $\pm 2,70$ & $\pm 1,56$ & $\pm 2,45$ & $\pm 2,05$ & $\pm 2,52$ \\
\hline \multirow[t]{2}{*}{$\mathrm{SaO}_{2}$} & GISO & 93,71 & 92,71 & $99,86 \mathrm{~A}$ & $100 \mathrm{~A}$ & $100 \mathrm{~A}$ & $100 \mathrm{~A}$ \\
\hline & & $\pm 1,97$ & $\pm 2,69$ & $\pm 0,35$ & \pm 0 & \pm 0 & \pm 0 \\
\hline \multirow[t]{2}{*}{$(\%)$} & GPRO & 94,57 & 93 & $100 \mathrm{~A}$ & $100 \mathrm{~A}$ & $100 \mathrm{~A}$ & $100 \mathrm{~A}$ \\
\hline & & $\pm 2,88$ & $\pm 1,83$ & \pm 0 & \pm 0 & \pm 0 & \pm 0 \\
\hline \multirow[t]{2}{*}{ Glicemia } & GISO & 86,2 & $137,5 \mathrm{Aa}$ & $156,5 \mathrm{~A}$ & $163,7 \mathrm{~A}$ & $145,5 \mathrm{~A}$ & $129,2 \mathrm{~A}$ \\
\hline & & $\pm 5,0$ & $\pm 24,1$ & $\pm 24,5$ & $\pm 31,1$ & $\pm 32,5$ & $\pm 29,2$ \\
\hline \multirow[t]{2}{*}{ (mg/dL) } & GPRO & 70,9 & $87,9 \mathrm{Ab}$ & $104,7 \mathrm{~A}$ & $110,9 \mathrm{~A}$ & $113,9 \mathrm{~A}$ & $103,9 \mathrm{~A}$ \\
\hline & & $\pm 9,2$ & $\pm 16,1$ & $\pm 22,5$ & $\pm 31,0$ & $\pm 37,7$ & $\pm 32,4$ \\
\hline
\end{tabular}

Letra A na linha indica valor diferente de M0, teste de Dunnet $(\mathrm{P} \leq 0,05)$. Letras minúsculas diferentes nas colunas, dentro de cada momento, indicam valor diferente entre grupos, Teste $t(P \leq 0,05)$.

Os tempos para extubação foram de 7,0土1,5 e $5,4 \pm 1,5$ minutos no GISO e GPRO, respectivamente, não havendo diferença significativa entre os grupos. Não foi observada ocorrência de sinais de excitação, vocalização ou qualquer intercorrência.

\section{DISCUSSÃO}

A dose de detomidina empregada no presente estudo (20mcg. $\mathrm{kg}^{-1} \mathrm{IV}$ ) pode ser considerada intermediária quando comparada à literatura, em que doses de 10 a $20 \mathrm{mcg} . \mathrm{kg}^{-1} \mathrm{IV}$ promovem sedação moderada, relaxamento muscular e leve ataxia (Celly et al., 1997; Kastner, 2006), enquanto $30 \mathrm{mcg} \cdot \mathrm{kg}^{-1}$ resultam em sedação intensa, severa ataxia e até mesmo decúbito (Trabach et al., 2011). A salivação profusa observada em todos os animais de ambos os grupos pode estar atribuída à diminuição do reflexo de deglutição, com acúmulo de saliva na cavidade bucal (Kastner, 2006).

Observou-se redução média de $40 \%$ da FC após a pré-medicação dos animais de ambos os grupos, corroborando os achados de Trabach et al. (2011), os quais utilizaram 30mcg. $\mathrm{kg}^{-1} \mathrm{IM}$ de detomidina em ovinos e relataram também uma redução de $40 \%$ nesse parâmetro. O efeito hipertensor desencadeado pelos alfa- 2 agonistas 
não foi observado, ao contrário do que observaram Celly et al. (1997), que demonstraram elevação de $25 \%$ da PAM em relação aos valores basais, cinco minutos após a administração de $30 \mathrm{mcg} \cdot \mathrm{kg}^{-1}$ de detomidina IV em ovinos. Presume-se que a morfina não tenha influenciado nessas variáveis discutidas, visto que Upton et al. (2003), ao utilizarem $0,6 \mathrm{mg} \cdot \mathrm{kg}^{-1}$ de morfina IV em ovinos, não observaram alterações quanto à $\mathrm{FC}$ e à pressão arterial.

A vasoconstrição arterial e principalmente no leito vascular venoso promovida pelos alfa-2 agonistas (Kastner, 2006) pode ser o fator desencadeante do aumento da pressão venosa central observada após a administração da detomidina, no presente estudo. Essa elevação e posterior redução corroboram Chitale (1998) em estudo com caprinos pré-medicados com diazepam associado à xilazina ou à romifidina.

Houve elevação da glicemia em todos os animais deste estudo, logo após a administração da detomidina. Esse efeito é desencadeado por aumento na produção de glicose pelo fígado e diminuição da secreção pancreática de insulina, reduzindo a utilização de glicose sanguínea pelos tecidos periféricos (Kastner, 2006).

O efeito diurético dos alfa-2 agonistas ocorre, principalmente, devido à diminuição da secreção do hormônio antidiurético (vasopressina), também podendo promover diurese hiperosmótica quando a taxa de filtração glomerular para glicose é excedida. No presente estudo, após a administração da detomidina, $64,3 \%$ dos animais (cinco de sete no GISO e quatro de sete do GPRO) apresentaram diurese alguns minutos após sua administração.

A indução no GPRO, utilizando-se $4 \mathrm{mg} \cdot \mathrm{kg}^{-1}$ de propofol, mostrou-se eficaz e segura, pois promoveu abolição dos reflexos laríngeos e de deglutição, bem como adequado relaxamento muscular, com ausência de sinais de dor ou excitação durante e após sua administração. Resultados semelhantes com a mesma dose indutora em ovinos já foram descritos (Kumari et al., 2013).

O propofol atualmente é o fármaco indutor mais utilizado em pequenos animais, promovendo rápida e suave indução anestésica, e vem ganhando uso também em pequenos ruminantes
(Galatos, 2011). No presente estudo, os valores de frequência cardíaca e pressões arteriais mantiveram-se reduzidos após a indução com propofol, em comparação ao momento basal, durante os primeiros momentos pós-indução anestésica, retornando próximos aos valores basais com o transcorrer do tempo, sempre dentro dos limites fisiológicos para a espécie. Nos estudos de Andaluz (2003), em que doses indutoras similares foram empregadas, observouse redução da frequência cardíaca.

Sabe-se que a redução da pressão arterial causada pelo propofol se deve à redução do débito cardíaco, do volume sistólico e, principalmente, a um decréscimo da resistência vascular sistêmica por inibição de mecanismos vasopressores medulares, levando à vasodilatação (Dzikiti, 2013).

Estudos demonstram que a depressão respiratória dose-dependente é uma característica comum da utilização de propofol, pela atuação direta sobre o centro respiratório do $\mathrm{SNC}$, sendo mais pronunciada quando esse fármaco é utilizado como agente único, em doses elevadas, e administrado rapidamente (Andaluz, 2003; Mohamadnia et al., 2008; Epstein, 2011). Correia et al. (1996), utilizando a mesma dose indutora empregada neste estudo, administrada também ao longo de 60 segundos, não relataram apneia após a indução dos animais, diferentemente do presente estudo, em que $14,3 \%$ (um de sete animais) do GPRO apresentaram apneia transitória, de período aproximado de 40 segundos, corroborando os achados de Mohamadnia et al. (2008).

A utilização de $0,5 \mathrm{mg} \cdot \mathrm{kg}^{-1}$ de diazepam associado a $5 \mathrm{mg} \cdot \mathrm{kg}^{-1}$ de cetamina IV no GISO manteve presentes os reflexos laríngeos e de deglutição em 43\% dos animais (3/7), mesmo após a administração de lidocaína spray sobre a laringe, mas não impossibilitou a intubação orotraqueal ou requereu doses suplementares, sendo apenas necessário um período mais prolongado para nova tentativa de intubação. Jud et al. (2010) relataram resultados similares, os quais descreveram facilidade durante a intubação orotraqueal ao compararem o uso de $0,1 \mathrm{mg} . \mathrm{kg}^{-1}$ de xilazina e $3 \mathrm{mg} . \mathrm{kg}^{-1}$ de cetamina racêmica ou $1,5 \mathrm{mg} . \mathrm{kg}^{-1}$ de cetamina $\mathrm{S}+$ em caprinos, mas que requereu mais de uma tentativa para intubação dos animais. A cetamina pode promover efeitos 
diretos e indiretos por meio do aumento do tônus simpático, aumento do DC, aumento da PAM, da estimulação direta do sistema nervoso central (SNC), do efeito inotrópico positivo sobre o miocárdio e do efeito no bloqueio da recaptação de catecolaminas (Dzikiti, 2013).

Embora este protocolo de indução seja amplamente utilizado e até mesmo indicado para pacientes críticos por desencadear mínimos efeitos cardiorrespiratórios (Abrahamsen, 2013), observou-se a ocorrência de hipercapnia no momento pós-indução, com níveis de $\mathrm{PaCO}_{2}$ maiores que $50 \mathrm{mmHg}$ em cinco dos sete animais, corroborando os achados de Ismail et al. (2010) em ovinos anestesiados com $0,1 \mathrm{mg} \cdot \mathrm{kg}^{-1}$ de xilazina, $0,25 \mathrm{mg} \cdot \mathrm{kg}^{-1}$ de diazepam e $5 \mathrm{mg} \cdot \mathrm{kg}^{-1}$ de cetamina IV.

Os valores de $\mathrm{PaO}_{2}$ devem estar próximos de quatro a cinco vezes a fração inspirada de oxigênio fornecida ao paciente, contudo a mecânica ventilatória em pequenos ruminantes pode ser diretamente influenciada pelo decúbito a que o animal é submetido (Mogoa et al., 2000), refletindo-se no fato de os valores basais médios estarem abaixo do esperado para a $\mathrm{PaO}_{2}$ nos animais do presente estudo. A maior desvantagem do emprego de alfa-2 adrenérgicos em ovinos é a possibilidade do desenvolvimento de hipoxemia e edema pulmonar (Celly et al., 1997), embora os valores pós-MPA não tenham sido menores quando comparados aos valores basais. Com relação aos valores de $\mathrm{pH}, \mathrm{BE}$ e $\mathrm{HCO}_{-3}$, observou-se a ocorrência de leve alcalose metabólica pós-indução anestésica, podendo estar atrelada à hipoventilação, sendo tentativa compensatória diante da elevação dos valores de $\mathrm{PaCO}_{2}$. A instituição de ventilação mecânica mostrou-se eficaz e adequada ao serem observados os parâmetros $\mathrm{PaCO}_{2}$ e $\mathrm{SaO}_{2}$. Embora tenha ocorrido normalização dos valores de $\mathrm{PaCO}_{2}$ após instituição da ventilação mecânica, o acúmulo de bicarbonato ainda se manteve presente, podendo esse fato ser explicado pela alteração da filtração e pelo clearance renal desencadeado pelos efeitos alfa adrenérgicos dos alfa- 2 agonistas, com aumento para eliminação de íons cloro e acúmulo de íons bicarbonato, mas sem alterações para eliminação de íons hidrogênio (Burton et al., 1998).

Os efeitos depressores do propofol refletiram-se na redução das pressões arteriais em torno de
$20 \%$ após instituição da infusão contínua, corroborando o estudo de Andaluz (2003), que, ao utilizar $0,4 \mathrm{mg} \cdot \mathrm{kg}^{-1} \cdot \mathrm{min}^{-1} \mathrm{em}$ ovinos, observou redução da PAM em torno de 25\% 15 minutos após o início da infusão contínua. A redução da taxa de propofol para $0,2 \mathrm{mg} \cdot \mathrm{kg}^{-1} \cdot \mathrm{min}^{-1}$ durante a anestesia propiciou plano anestésico adequado e superficial. Ludbrook et al. (1999), ao avaliarem a farmacocinética do propofol em ovinos, relataram sedação intensa ou plano anestésico superficial nos animais, sendo que estes animais não foram induzidos à anestesia com o propofol, sendo apenas instituída uma taxa de $0,2 \mathrm{mg} . \mathrm{kg}^{-}$ ${ }^{1} . \mathrm{min}^{-1}$. Presume-se que o efeito sinérgico das demais medicações e a eficácia do bloqueio subaracnoideo propiciaram reduzir a taxa inicial de infusão.

Entre os anestésicos inalatórios, o isoflurano é o agente que promove maior redução dos valores da pressão arterial, sendo esse efeito atribuído à redução na resistência vascular periférica e discreta redução no DC (Hikasa et al., 1998). No estudo em questão, após início da manutenção com isoflurano, os valores de $\mathrm{FC}$ e pressões arteriais mantiveram-se estáveis. Dzikiti et al. (2013), em estudo com a espécie caprina, e Mattos-Júnior (2012), em estudo com a espécie ovina, empregaram o isoflurano de forma isolada na dose de 1 CAM e relataram estabilidade cardiovascular, referente a FC, pressões arteriais, PVC e DC, sem alterações significativas desses parâmetros.

Ao se compararem valores de CAM do isoflurano de $1,31 \mathrm{~V} \%$ determinados por MattosJúnior (2012), pôde-se inferir que a MPA com detomidina e morfina e o protocolo indutor cetamina/diazepam e um bloqueio subaracnoideo eficaz tiveram efeito sinérgico neste estudo, pois os valores médios para manutenção dos animais foram $50 \%$ menores, ou seja, $1 / 2$ CAM foi necessário para manutenção adequada do plano anestésico.

O protocolo de anestesia subaracnoidea mostrouse eficaz em $100 \%$ dos animais de ambos os grupos, pois alterações cardiovasculares não foram observadas durante os momentos de incisão de pele, periósteo e, principalmente, durante a realização das osteotomias, sendo considerado o estímulo supramáximo do procedimento cirúrgico. A dose utilizada neste estudo foi embasada nos estudos de Moreira 
(2012), o qual relatou latência quase imediata após a administração de ropivacaína em ovinos conscientes, a qual proporcionou um bloqueio sensitivo de aproximadamente 166 minutos. Diante desses resultados citados, o período cirúrgico teve anestesia garantida, pois o tempo cirúrgico foi menor do que o bloqueio sensitivo promovido pela ropivacaína.

O tempo médio para extubação encontrado no GISO é semelhante aos dos estudos em que ovinos foram sedados com xilazina, induzidos com cetamina e midazolam e mantidos com isoflurano (Mohamadnia et al., 2008) ou prémedicados com medetomidina e induzidos e mantidos com isoflurano (Vettorato et al., 2012), mas são maiores do que quando o isoflurano foi empregado de forma isolada em ovinos não prémedicados, que apresentaram período médio de 3,27 minutos (Mattos-Júnior, 2012). A rápida recuperação anestésica promovida por agentes inalatórios está atrelada a suas características farmacológicas, como coeficiente solubilidade sangue/gás, e à sua eliminação quase que totalmente pela via pulmonar, com mínima metabolização hepática e ausência de efeito cumulativo (Alibhai, 2001).

O perfil farmacocinético do propofol em ovinos, caracterizado por alto volume de distribuição, rápido metabolismo e alta taxa de eliminação, tanto em dose bolus quanto em infusão contínua, propicia rápida recuperação anestésica (Weaver et al., 2001).

Correia et al. (1996) relataram período para extubação de 2,8 minutos em ovinos submetidos à infusão isolada de propofol, e Mattos-Júnior et al., 2013 relataram tempo médio de 9,3 e 11,3 em ovinos mantidos sob anestesia geral com propofol-fentanil ou com propofol-fentanillidocaína, respectivamente. Nota-se a influência de fármacos adjuvantes em prolongar o período de recuperação dos animais.

\section{CONCLUSÃO}

A manutenção anestésica com isoflurano ou propofol, associada à anestesia subaracnoidea com ropivacaína/morfina, promoveram plano anestésico similar, com mínimos efeitos cardiovasculares ou hemogasométricos.

\section{REFERÊNCIAS}

ABRAHAMSEN, E.J. Chemical restraint and injectable anesthesia of ruminants. Vet Clin. N. Am. Food Anim. Pract., v.29, p.209-227, 2013.

ABU-AHMED, H. Sedative and hematobiochemical effects of midazolam and midazolam-ketamine combination in Baladi Goats. GlobalVet., v.10, p.742-747, 2013.

ADAMS, D.; MCKINLEY, M. The sheep. Anzccart Hum. Sci., v.9, p.1-13, 2009.

ALIBHAI, H.I.K. Aspects of inhalation anaesthesia in the goat. 2001. Nf. Thesis (PhD) - University of London, London.

ANDALUZ, A. Administración de propofol (2,6diisopropilfenol) en ovejas gestantes a término: estúdio del passo placentário, farmacocinética y efectos cardiovasculares, respiratórios y del equilíbrio ácido-base em las madres y sus fetos. 2003. 139f. Tesis (Doutoral) - Dacultat de Veterinaria, Universitat Autónoma de Barcelona, Barcelona.

BURTON, S.; LEMKE, K.A.; IHLE, S.L.; MACKENZIE, A.L. Effects of medetomidine on serum osmolality, urine volume, oslmolality and $\mathrm{pH}$; free water clearance; and fractional clearance of sodium, chloride, potassium, and glucose in dogs. Am. J. Vet. Res., v.59, p.756$761,1998$.

CELLY, C.S.; McDONELL, W.N.; YOUNG, S.S.; BLACK, W.D. The comparative hypoxaemic of four a2 adrenoceptor agonists (xylazine, romifidine, detomidine and medetomidine) in sheep. J. Vet. Pharmacol. Ther., v.20, p.464-471, 1997.

CHITALE, D.; PRATAP, K.J.; AMARPAL, A. et al. Alpha2 agonists with diazepam as preanaesthetic to ketamine anaesthesia in goats: haemodynamic effects. Indian J. Anim. Sci., v.68, p.1138-1139, 1998.

CORREIA, D.; NOLAN, A.M.; REID, J. Pharmacokinetics of propofol infusions, either alone or with ketamine, in sheep premedicated with acepromazine and papaveretum. Res. Vet. Sci., v.60, p.213-217, 1996. 
DEROSSI, R.; PAGLIOSA, R.; MÔDOLO, T.C. et al. Thoracic epidural analgesia via the lumbosacral approach using multiport catheters with a low concentration of bupivacaine and morphine in sheep. Vet. Anaest. Analg., v.39, p.306-314, 2012.

DZIKITI, T.B. Intravenous anaesthesia in goats: a review. J. South Afr. Vet. Assoc., v.84, p.1-8, 2013.

EPSTEIN, A. Effects of general anesthesia on respiratory system. Israel J. Vet. Med., v.66, p.913, 2011.

GALATOS, A.D. Anesthesia and analgesia in sheep and goats. Vet. Clin. N. Am.: Food Animal Pract., v.27, p.47-59, 2011.

HIKASA, Y.; OKUYAMA, K.; KAKUTA, T. et al. Anesthetic potency and cardiopulmonary effects of sevoflurane in goats: comparasion with isoflurane and halothane. Can. J. Vet. Res., v.63, p.205-210, 1998.

ISMAIL, Z.B.; JAWASREH, K.; AL-MAJALI. Effect of xylazine-ketamine-diazepam anesthesia on certain clinical and arterial blood gas parameters in sheep and goats. Comp. Clin. Pathol., v.19, p.11-14, 2010.

JUD, R.; PICEK, S.; MAKARA, M.A. et al. Comparasion of racemic and S-ketamine agents for induction of anaesthesia in goats. Vet. Anaest. Analg., v.37, p.511-518, 2010.

KASTNER, S. A $\mathrm{A}_{2}$-agonists in sheep: a review. Vet. Anaest. Analg., v.33, p.79-96, 2006.

KUMARI, V.R.; MADAN, A.K.; DAS, A.K.; JADON, N.S. Total intravenous anaesthesia (TIVA) with propofol in sheep: A clinical and haematobiochemical study. Indian J. Vet. Surg., v.34, p.32-34, 2013.

LIZARRAGA, I.; CHAMBERS, J.P. Use of analgesic drugs for pain managment in sheep. New Zeal. Vet. J., v.60, p.87-94, 2012.

LUDBROOK, G.L.; UPTON, R.N.; GRANT, C.; MARTINEZ, A. A Compartmental analysis of the pharmacokinetics of propofol in sheep. $J$. Pharmacol. Biopharma., v.27, p.329-338, 1999.
MATTOS-JUNIOR, E.; MINERVINO, A.H.H.; BARRÊTO-JUNIOR, R.A. et al. High doses of lidocaine as a constant rate infusion in propofol/fentanyl anaesthetized sheep: cardiorespiratory effects. Semina: Ciênc. Agr., v.34, p.323-334, 2013.

MATTOS-JUNIOR. Anestesia inalatória em ovinos: estudo comparativo entre o halotano, isofluorano e sevofluorano. 2012. 120f. Tese (Doutorado em Ciências)-Faculdade de Medicina Veterinária e Zootecnia, Universidade de São Paulo, São Paulo, SP.

MOGOA, E.G.; STEGMANN, G.F.; GUTHIRE, A.J. Effects of xylazine on acid-base and arterial blood-gas tensions in goats under different environmental temperature and humidity conditions. J. S. Afr. Vet. Assoc., v.71, p.229231,2000 .

MOHAMADNIA, A.R.; SABERIN, L.; SHAHROKHI, M. et al. Evaluation of food withholding time on propofol total intravenous anestesia in sheep. Iranian J. Vet. Surg., v.3, p.61-69, 2008.

MOREIRA, R.A. Avaliação anestésica e efeitos cardiorrespiratórios da administração subaracnoidéa de ropivacaína isolada ou em associação à metadona em ovinos. 2012. 69f. Dissertação (Mestrado) - Faculdade de Agronomia e Medicina Veterinária, Universidade de Brasília, Brasília, DF.

TRABACH, T.C.; BARROS, R.J.S.; MENDES, V.R.A. et al. Avaliação clínica do cloridrato de detomidina em ovinos. Pubvet, v.5, np., 2011.

UPTON, R.N.; LUDBROOK, G.L.; MARTINEZ, A.M. et al. Cerebral and lung kinetics of morphine in conscious sheep after short intravenous infusions. Lab. Investig., v.90, p.750-758, 2003.

VETTORATO, E.; SCHOFFMANN， G.; BURKE, J.G. et al. Clinical effects of isoflurane and sevoflurane in lambs. Vet. Anaest. Analg., v.39, p.495-502, 2012.

WEAVER, B.M.Q.; STADDON, G.E.; MAPLESON, W.W. Tissue/blood and tissue/ water partition coefficients for propofol in sheep. Brit. J. Anaest., v.86, p.693-703, 2001. 